\title{
Implementation of secondary fracture prevention services after hip fracture: a qualitative study using extended Normalization Process Theory
}

Sarah Drew ${ }^{1 *}$, Andrew Judge ${ }^{1,2}$, Carl May $^{3}$, Andrew Farmer ${ }^{4}$, Cyrus Cooper $^{1,2}$, M Kassim Javaid $^{1,2}$, Rachael Gooberman-Hill ${ }^{5}$ and The REFReSH study group

\begin{abstract}
Background: National and international guidance emphasizes the need for hospitals to have effective secondary fracture prevention services, to reduce the risk of future fractures in hip fracture patients. Variation exists in how hospitals organize these services, and there remain significant gaps in care. No research has systematically explored reasons for this to understand how to successfully implement these services. The objective of this study was to use extended Normalization Process Theory to understand how secondary fracture prevention services can be successfully implemented.
\end{abstract}

Methods: Forty-three semi-structured interviews were conducted with healthcare professionals involved in delivering secondary fracture prevention within 11 hospitals that receive patients with acute hip fracture in one region in England. These included orthogeriatricians, fracture prevention nurses and service managers. Extended Normalization Process Theory was used to inform study design and analysis.

Results: Extended Normalization Process Theory specifies four constructs relating to collective action in service implementation: capacity, potential, capability and contribution. The capacity of healthcare professionals to co-operate and co-ordinate their actions was achieved using dedicated fracture prevention co-ordinators to organize important processes of care. However, participants described effective communication with GPs as challenging. Individual potential and commitment to operationalize services was generally high. Shared commitments were promoted through multi-disciplinary team working, facilitated by fracture prevention co-ordinators. Healthcare professionals had capacity to deliver multiple components of services when co-ordinators 'freed up' time. As key agents in its intervention, fracture prevention coordinators were therefore indispensable to effective implementation.

Aside from difficulty of co-ordination with primary care, the intervention was highly workable and easily integrated into practice. Nevertheless, implementation was threatened by under-staffed and under-resourced services, lack of capacity to administer scans and poor patient access. To ensure ongoing service delivery, the contributions of healthcare professionals were shaped by planning, in multi-disciplinary team meetings, the use of clinical databases to identify patients and define the composition of clinical work and monitoring to improve clinical practice.

Conclusions: Findings identify and describe elements needed to implement secondary fracture prevention services successfully. The study highlights the value of Normalization Process Theory to achieve comprehensive understanding of healthcare professionals' experiences in enacting a complex intervention.

Keywords: Implementation, Normalization Process Theory, Qualitative research, Osteoporosis, Fragility fracture, Hip fracture

\footnotetext{
* Correspondence: sarah.drew@ndorms.ox.ac.uk

${ }^{1}$ Nuffield Department of Orthopaedics, Rheumatology and Musculoskeletal Sciences, Oxford NIHR Musculoskeletal Biomedical Research Unit, University of Oxford, Windmill Road, Headington, Oxford OX3 7LD, UK

Full list of author information is available at the end of the article
} 


\section{Background}

Hip fractures present an important public health problem. Each year, 87,000 hip fractures occur annually in the UK [1] with a cost (including medical and social care) of around $£ 2.3$ billion a year [2]. Hip fractures usually occur when individuals with underlying osteoporosis fall $[3,2]$. These patients are a high risk of further fractures and premature death [4-6]. The risk of second hip fracture ranges from $2.3 \%$ to $10.6 \%[7,8]$ and mortality during the first year after fracture ranges from $8.4 \%$ to $36 \%$ [4]. Effective management of these patients can significantly reduce this risk, which is why professional bodies have produced comprehensive guidance about the management of hip fracture [1,9-12]. Fracture prevention services should have four main components: case finding those at risk of further fractures; undertaking an evidence-based osteoporosis assessment; treatment initiation in accordance with guidelines for both bone health and falls risk reduction and then strategies to monitor and improve adherence to recommended therapies [12]. Since the provision of these services is multi-disciplinary [1], guidance recommends structuring services around a dedicated co-ordinator who provides a link between all the multi-disciplinary teams involved in fracture prevention [13], an approach known as a Fracture Liaison Service [14]. However, considerable gaps in patient care following fracture still exist [15] with marked variation in how services are delivered locally $[16,17]$, and it is unclear how best to implement these services.

The implementation of interventions is increasingly being studied using theory-led research designs $[18,19]$. In this study, we used extended Normalization Process Theory [20] as a theoretical framework because it characterizes and explains the collective actions of participants (patient, professionals and others) in the implementation of new ways of enacting and organizing health care. Such an approach is a useful counterpoint to perspectives on the transmission and stabilization of innovations through networks $[21,22]$ and theories of individual differences in behavioural change [23,24], because it attends to the factors that promote or inhibit the kinds of co-operative work that is needed to implement service innovations in complex organizational contexts. The theory builds on previous iterations of Normalization Process Theory that have presented implementation as a social process. Extended Normalization Process Theory incorporates psychological and network perspectives into earlier iterations of Normalization Process Theory to build a more comprehensive model to explain phenomena. Previous iterations were originally used to explore professional action in the implementation of interventions in telehealth and e-health [25-27], but their application has since broadened to include a range of services across primary and secondary care [28-31]. The theories are also increasingly being applied to care settings from outside of the UK $[32,33,28]$. However, to our knowledge, this is the first study to use extended Normalization Process Theory.

The aim of this study was to use extended Normalization Process Theory to understand how and why secondary fracture prevention services can be successfully implemented in secondary care. This serves to inform the implementation and integration of these services into practice. In addition, as one of the first studies to use extended Normalization Process Theory, there was the potential to learn about the mechanics of its application in research.

\section{Methods}

\section{Sample}

A purposive sample was designed to include a range of healthcare professionals from all 11 Acute NHS Trusts in a region in England who met the criteria of working in secondary care and with experience and knowledge of secondary fracture prevention after hip fracture [34]. The sample included orthogeriatricians, fracture prevention nurses, trauma nurses, hospital practitioners in osteoporosis, surgeons and service managers.

Potential participants were identified by the clinical lead/champion in osteoporosis and an operational service manager in trauma who were both working within the region. In three waves of recruitment, the study team approached potential participants by email, followed up by reminder emails when appropriate 2 weeks later. In some cases, this was then followed up by a telephone call. We also used snowball sampling [35] such that participants recommended other healthcare professionals who they knew were also involved in fracture prevention. In total, 82 healthcare professionals were contacted to take part in the study. Of these, 43 agreed to take part. The remainder either declined or were unavailable. Rather than aiming to achieve saturation [36], the final sample size reflects our aim of conducting criterion sampling to include an appropriate range of professionals to enable us to address the research question in light of extended Normalization Process Theory and their availability [37]. This meant prioritising the recruitment of professionals most familiar with the processes of implementing fracture prevention services and ensuring participants were adequately drawn from each of the 11 hospitals.

\section{Ethical approval}

Ethical approval was provided by the University of Oxford's Central University Research Ethics Committee (CUREC) in 2012, reference number MSD-IDREC-C12012-147. Written informed consent was provided by all participants prior to interview. This confirmed that participants understood that their participation was voluntary 
and that they were willing to let the project researcher (SD) include anonymous quotations from them in the write up of the study. Each involved NHS Trust provided R\&D approval.

\section{Interview procedure}

A qualitative researcher (SD) undertook face-to-face interviews in 2013 with one interview conducted by telephone. Interviews were between 30 and 50 min long. A topic guide divided into two themes was used to inform interview questions (Additional file 1). Theme 1 was based on the four core elements of a fracture prevention service outlined by the International Osteoporosis Foundation (IOF) as part of the 'Capture the Fracture' initiative [12]. This helped the researcher to explore participants' views on the best models of care across the four main components of a fracture prevention service and coordination of care. Theme 2 was structured around the four constructs of extended Normalization Process Theory to enable exploration of participants' experiences of implementing fracture prevention services (Table 1). Participants were also encouraged to raise any issues that they thought relevant, and the interviewer used methods such as 'probing' to help achieve depth [38].

\section{Extended Normalization Process Theory}

The implementation of interventions is dependent on the ability of participants to fulfil four criteria which can be understood using four constructs [20], as outlined below:

\section{Data analysis}

Interviews were audio-recorded, transcribed, anonymised and imported into the qualitative data analysis software NVivo. An abductive analysis [39] was conducted. This involved coding data inductively using a thematic analysis, and all data were scrutinised for relevance to implementation. To relate the data to extended Normalization Process Theory, codes were then transposed onto the four main constructs of the theory. Data was then displayed on charts using the framework approach to data organization [40]. Twenty percent of the interview transcripts were independently coded by another member of the team (RG-H), and codes compared and discussed to arrive at a single code list which was refined as the analysis progressed [41]. Descriptive accounts of the data were then generated.

\section{Results}

\section{Characteristics of sample}

The 43 participants comprised eight fracture prevention nurses, four orthogeriatricians, four geriatricians, two GP osteoporosis specialists, five consultant trauma orthopaedic surgeons, eight rheumatology consultants, additional nursing staff, one falls co-ordinator, one falls nurse, one bone densitometry specialist and five service managers. Between three and seven were drawn from each of the 11 hospitals. Time spent working at the hospital ranged between 1 and 27 years and years of experience since qualification in their current role ranged between 8 months to 32 years.

A summary of the codes identified and their relation to the four main constructs of extended Normalization Process Theory is outlined in Table 2.

Below, we explore healthcare professionals' experiences and views about issues that affect the implementation of services to prevent secondary fractures after hip fracture using the four constructs of extended Normalization Process Theory in more detail.

\section{Capacity}

Implementing a fracture prevention service depends on participants' capacity to co-operate and co-ordinate their actions.

Participants in this intervention made significant personal investments in building co-operative capacity. However, their support for the intervention was focused through the allocation of a dedicated fracture prevention co-ordinator who both organized important processes of care and supported the complex relationships between other participants. This support was manifest in two kinds of work. First, it was manifest in 'multi-disciplinary paperwork': the protocols and proforma records that gave structure to particular parts of the care process and which could be easily understood and shared between different professional groups. Second, it was present in support for multi-disciplinary teamwork that meant that discussions and decisions about policy and patients could be undertaken co-operatively and in the open.

The visibility of these two kinds of support meant that a culture of co-operation developed around the

Table 1 The four constructs of extended Normalization Process Theory

\begin{tabular}{ll}
\hline Construct & Description \\
\hline 'Capacity' & Implementing an intervention depends on participants' capacity to co-operate and co-ordinate their actions \\
'Potential' & Translating capacity into action depends on participants' commitment to operationalize the intervention \\
'Capability' & The capability of participants to enact the intervention depends on its workability and integration into everyday practice \\
'Contribution' & The implementation of an intervention over time depends on participants' contributions to enacting it by investing in meaning, \\
& commitment, effort and appraisal
\end{tabular}


Table 2 Codes identified and their relation to the four main constructs of extended Normalization Process Theory

\begin{tabular}{|c|c|c|c|}
\hline “Capacity' & ‘Potential' & ‘Capability' & ‘Contribution' \\
\hline $\begin{array}{l}\text { Role of dedicated fracture prevention } \\
\text { coordinator }\end{array}$ & $\begin{array}{l}\text { High levels of support for } \\
\text { introducing service }\end{array}$ & $\begin{array}{l}\text { Fracture prevention coordinators 'freeing } \\
\text { up' professionals previously engaged in care }\end{array}$ & $\begin{array}{l}\text { Multi-disciplinary team } \\
\text { meetings }\end{array}$ \\
\hline $\begin{array}{l}\text { Multi-disciplinary paperwork: protocols and } \\
\text { proforma records }\end{array}$ & $\begin{array}{l}\text { Lack of support for introducing } \\
\text { service from some professionals }\end{array}$ & Lack of time to deliver intervention & Clinical databases \\
\hline $\begin{array}{l}\text { Multi-disciplinary team-work: multi- } \\
\text { disciplinary team meetings, joint ward } \\
\text { rounds }\end{array}$ & $\begin{array}{l}\text { Relationships between different } \\
\text { professional groups }\end{array}$ & Lack of capacity to administer DXA scans & $\begin{array}{l}\text { Internal monitoring } \\
\text { systems }\end{array}$ \\
\hline Positive working relationships & Multi-disciplinary team working & $\begin{array}{l}\text { Challenges faced by service users in } \\
\text { accessing services }\end{array}$ & $\begin{array}{l}\text { External monitoring } \\
\text { systems linked to } \\
\text { funding }\end{array}$ \\
\hline $\begin{array}{l}\text { Location of professionals close to the } \\
\text { service and each other }\end{array}$ & $\begin{array}{l}\text { Role of fracture prevention } \\
\text { coordinator }\end{array}$ & & \\
\hline $\begin{array}{l}\text { Challenge of securing co-operation and } \\
\text { communication with GPs }\end{array}$ & $\begin{array}{l}\text { Varying commitment from } \\
\text { practitioners in primary care }\end{array}$ & & \\
\hline \multicolumn{4}{|l|}{$\begin{array}{l}\text { High workload in primary care impacting } \\
\text { on time spent implementing intervention }\end{array}$} \\
\hline \multicolumn{4}{|l|}{$\begin{array}{l}\text { Written communication with GPs, } \\
\text { especially discharge summaries and DXA } \\
\text { reports }\end{array}$} \\
\hline $\begin{array}{l}\text { Potential role of fracture prevention } \\
\text { coordinators in primary care }\end{array}$ & & & \\
\hline
\end{tabular}

intervention. This culture depended on shared expertise, as well as belonging to a co-operative group. When professionals felt closer to the centre of the service-close to the physical location and with more involvement in implementation-then they experienced better communication and enhanced enthusiasm. A key problem for participants was therefore securing the co-operation and interest of GPs. They saw this as very variable, depending not only on the extent of the GPs experience and interest in fracture prevention but also on structural factors to do with primary care workload and reimbursement. Responding to these perceived difficulties involved attempting to improve written communications-especially discharge summaries and dual energy x-ray absorptiometry (DXA) reports measuring bone density. Participants invested a good deal of effort in these activities but recognized that they were not necessarily the most effective mechanism for informing GPs. They saw a solution in extending the fracture prevention co-ordinator role into the community through a cadre of specialist nurses that could work with GPs to improve the quality of clinical care (Additional file 2).

\section{Potential}

Translating capacity into action depends on participants' commitment to operationalize the fracture prevention service.

Participants in this study were mainly enthusiastic and committed to enacting the intervention. They agreed that the fracture prevention service was necessary, and they were highly supportive of the service in action.
Those that were not tended to be characterized by their peers as negative or unsupportive personalities, but it was clear that this lack of support-sometimes manifest in open obstruction from a small number of clinicianswas a problem that reflected other longstanding complexities in relations between different specialists and within hospital departments. These individual differences are common problems but did not present insuperable difficulties and were overcome because of the shared commitments that developed through co-operative and multidisciplinary working that the service seemed to inspire. However, that multi-disciplinary culture of co-operation needed to be built and maintained. Some participants were clear that responsibility for sustaining shared commitments was owned by the fracture prevention coordinator and that a central part of the co-ordinator's role was to glue together different professional cultures and ensure that there was constant agreement between them about the goals of the service. Once again, this meant that there were complexities at the margins and that the perceived divide between the hospital service and primary care was a very visible one, and GPs were seen as disengaged from the work of fracture prevention (Additional file 3).

\section{Capability}

The capability of users to enact the components of a fracture prevention service depends on their potential for workability and integration in their everyday practice.

We have already emphasised that participants were enthusiastic about introducing the fracture prevention 
service because it introduced dedicated specialist nurses who delivered a new layer of service provision that centred on co-ordination and support, but at the same time, freed up the capacity of others who had previously been engaged in this work. In this sense, the service intervention was apparently highly workable and easily integrated into practice. But there were also important problems. These focused on three kinds of inequality. First, there were inequalities of attention. Participants characterized the settings in which they worked as under-staffed and underresourced, and this reduced the time that could be committed to patients but also, and just as important, to do the administrative and communications work that is necessary to ensure the effective operation of a clinical service. Second, there were inequalities of equipment. Participants pointed to the lack of capacity to administer DXA scans that arose from a small number of scanners operated by equally small numbers of staff. Finally, there were inequalities of patient access, participants pointed to the difficulties that some service users faced, because of poor coverage of public transport that some socio-economically disadvantaged users needed to get to the service in the first place (Additional file 4).

\section{Contribution}

Participants' contributions to enacting a fracture prevention service depend on them investing in meaning, commitment, effort and appraisal.

It is important to note that the introduction of a fracture prevention co-ordinator into these clinical services did not change the content of clinical work, but rather aimed to change its organizational structure and service delivery. We have discussed above how this seemed to involve important cultural changes in relations between different professions and specialisms, but it also involved some important changes in the ways that these groups made sense of fractures as an operational problem.

The introduction of a co-ordinator effected the explicit differentiation not only of the task of organization but also of the new ways to respond to it. Planning-through multi-disciplinary team meetings-seems to have become an important mechanism by which health professionals turned their potential and capacity to deliver the new service configuration into a coherent process and enrolled others into it. Clinical databases of different kinds were an important tool through which this was accomplished, since they specified individual beneficiaries of the service but also defined the level and composition of the whole clinical workload. Poor quality data was an obstacle to this, not simply because it interfered with identifying candidate patients and delivering clinical practice, but because it interfered with monitoring the outcomes of the work, both for individual patients and for healthcare providers. Monitoring was central to the enterprise and took two forms. First, it involved understanding clinical activity as a dynamic process within the hospital, attempting to understand the degree of continued bone protection therapy after patient discharge and attempting to appraise patient compliance with those therapies. Second, it involved linking clinical activity to funding mechanisms-whether this was the Best Practice Tariff in hospitals (where it seemed to deliver significant financial benefits) or the Quality Outcomes Framework in primary care (where it did not). Here, monitoring was seen as an important mechanism to bring about change, because it focused on the dynamic performance of service providers and the degree to which they could operationalize their shared goals (Additional file 5).

\section{Discussion}

\section{Principal findings}

This study has identified and explored healthcare professionals' experiences and views about issues that affect the implementation of secondary fracture prevention services using extended Normalization Process Theory [20]. Results showed that health professionals' capacity to cooperate and co-ordinate their actions were achieved by using dedicated fracture prevention co-ordinators who organized important processes of care. Effective communication with GPs was seen as challenging and participants advocated improvements in written communication whilst recognising that this had its limitations. The potential and commitment of health professionals to operationalize secondary fracture prevention services was generally high. Shared commitment to operationalisation was promoted through multi-disciplinary team working, facilitated by fracture prevention co-ordinators. However, healthcare professionals in secondary care saw the commitments of GPs as more variable. Health professionals' capability to enact the components of fracture prevention services was enhanced by the presence of fracture prevention coordinators who 'freed up' other healthcare professionals. As key agents in its intervention, fracture prevention coordinators were therefore indispensable to effective implementation. Aside from the challenges of cooperation and communication with primary care, fracture prevention services were seen as highly workable and easily integrated into working practice. Nevertheless, inequalities in attention to patients, equipment and patient access posed serious threats to successful implementation. To deliver the service over time, health professionals' contributions were shaped by effective planning in multi-disciplinary team meetings, the use of clinical databases to accomplish patient care and monitoring to improve clinical practice. 


\section{Relationship to current literature}

This study contributes to the existing body of work to identify the best models of care for the prevention of secondary fractures after hip fracture by exploring how these services are best implemented in practice. Previous research has highlighted the challenge of coordinating the multi-disciplinary teams involved in fracture prevention, and there is an international consensus about the need for co-ordinator-based models of care [12,13,42-44]. To promote the integration of primary and secondary care, the British Orthopaedic Association recommends ensuring that GPs receive comprehensive discharge summaries and recommendations alongside introduction of fracture prevention champions into primary care [1]. These strategies were also advocated by participants in the study described here. The potential value of introducing clinical databases and collecting and utilising audit data and cost-saving incentives has also been highlighted [44]. This study provides evidence of how these recommendations may be implemented in working practice within the NHS. In addition, the study identifies and describes the importance of potential and commitment of healthcare professionals to operationalize fracture prevention services. It also highlights the value of fracture prevention co-ordinators, not just in coordinating and organizing care but also in sustaining the shared commitments of the multi-disciplinary team.

Findings from this study can be understood within the wider context of the NHS. A lack of capacity for healthcare professionals to co-operate and co-ordinate their actions, especially across primary and secondary care, is apparent in a number of other conditions [45-47] and remains a persistent problem for policy makers [48]. In addition, there are claims that inequalities in attention to patients, equipment and patient access exist across the NHS [49]. Recent efficiency savings may present a further challenge to this [50].

\section{Strengths and weaknesses of the study}

The use of qualitative methods enabled us to undertake a detailed exploration of the opinions and experiences of professionals. The sample size of 43 participants was relatively small given the variety of professions included in the study, but we are confident that the data generated was sufficient to provide appropriate and detailed analysis of the experience and views of professionals working in secondary care settings. We do not distinguish between the views of different groups of healthcare professionals working within the field of fracture prevention as we found that there was little difference in their perspectives. Within the interviews, participants were asked to describe previous experiences of service implementation, and such recall may be influenced by memory and subsequent experience. Participants were also asked to define reasons for delivery of services, and this means that they would refer to their views about the practice of others. This type of data does not incorporate the perspectives of those other health professionals. This particularly applies to views about primary care, and participants were reflecting on their experiences of interaction with primary care. Also, the study only took place in one region, and although it included all NHS Trusts in that region, further work may be needed to explore fracture prevention services in other areas. We invited all health professionals $(n=82)$ involved in service delivery to take part in the interviews. Of these, 43 took part. A potential limitation of the study is that those who declined participation were less amenable or involved in intervention delivery, but the study nonetheless garnered much information about positive and negative aspects of implementation from those professionals who took part.

The theory was found to be valuable in helping us to explore the research topic because the theory was able to account for all of the experiences and challenges encountered by healthcare professionals in implementing the intervention. A challenge in the application of extended Normalization Process Theory was the overlapping nature of the constructs, meaning that data could be coded into more than one construct. A decision was therefore made to code data into more than one construct where relevant. In addition, we sometimes found it hard to be certain that we were categorising data into the 'correct' construct. Both of these issues are consistent with the existing literature [28]. To mitigate this, the study researchers collaborated closely with each other throughout the process to make decisions about how to code the data, to arrive at an agreed code list and application of the list. There was also the potential for tension between undertaking an abductive approach [39] whilst ensuring the data was not 'forced' into predefined constructs. Coding the data inductively using a thematic analysis before transposing it onto the constructs of extended Normalization Process Theory helped to address this since we first inductively coded and scrutinised all data for issues relating to implementation before applying extended Normalization Process Theory.

\section{Further research}

As the study is focused solely on the perspectives of professionals working in secondary care, further work could explore experiences of engagement with fracture prevention services and service provision in primary care. This would offer a comprehensive, 'system-wide' perspective which would over arch the division between primary and secondary care. Further research could also explore the experiences of hip fracture patients and their significant others of accessing these services to add a 'patient 
centred' context to the implementation of these services. In addition, whilst the study focused on fracture prevention rather than falls prevention services, we acknowledge that these are interrelated [1] and further studies could employ implementation science to explore these in complement to this study.

\section{Conclusions}

The study has successfully used extended Normalization Process Theory [20] to analyse the experiences of healthcare professionals when they work to enact a complex intervention. Identifying issues that impact on the implementation of facture prevention services after hip fracture provides information to healthcare professionals and service managers on how best to implement services for patients in the future. In addition, by using the most recent iteration of Normalization Process Theory [20], this study builds the evidence base that makes use of implementation science.

\section{Additional files}

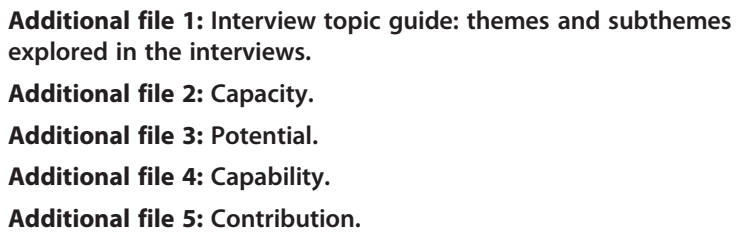

\section{Competing interests}

AJ has received consultancy, lecture fees and honoraria from Servier, UK Renal Registry, Oxford Craniofacial Unit, IDIAP Jordi Gol, Freshfields Bruckhaus Deringer, has held advisory board positions (which involved receipt of fees) from Anthera Pharmaceuticals, INC. and received consortium research grants from ROCHE. MKJ has received in the last 5 years honoraria for travel expenses, speaker fees and/or advisory committees from Lilly UK, Amgen, Servier, Merck, Medtronic, Internis and Consilient Health. He also serves on the Scientific Committee of the National Osteoporosis Society and International Osteoporosis Foundation. CC has received consultancy, lecture fees and honoraria from AMGEN, GSK, Alliance for Better Bone Health, MSD, Eli Lilly, Pfizer, Novartis, Servier, Medtronic and Roche. SD, CM, AF and RGH declare that they have no competing interests.

\section{Authors' contributions \\ AJ, RGH, MKJ, CC, AF and all of The REFReSH Study Group contributed to the design of this research. SD, RGH, CM, CC, MKJ and AJ all contributed to the acquisition, analysis or interpretation of data. RGH is the guarantor. All authors contributed to drafting this work and revising it for important intellectual content, and all gave final approval for the version to be submitted.}

\section{Acknowledgements}

The ReFRESH study group consists of Dr Andrew David Judge, Dr Muhammad Kassim Javaid, Professor Nigel Arden, Professor Cyrus Cooper, Professor Andrew Farmer, Dr Daniel Prieto-Alhambra, Dr Jose Leal, Professor Michael Goldacre, Professor Alastair Gray, Dr Janet Lippett, Dr Rachael Gooberman-Hill and Laura Graham.

\section{HS\&DR Funding}

This project was funded by the National Institute for Health Research Health Services and Delivery Research Programme (project number 11/1023/01). We also wish to acknowledge the NIHR Musculoskeletal Biomedical Research Unit for financial support.

\section{Department of Health Disclaimer}

The views and opinions expressed therein are those of the authors and do not necessarily reflect those of the HS\&DR Programme, NIHR, NHS or the Department of Health.

\section{Author details}

${ }^{1}$ Nuffield Department of Orthopaedics, Rheumatology and Musculoskeletal Sciences, Oxford NIHR Musculoskeletal Biomedical Research Unit, University of Oxford, Windmill Road, Headington, Oxford OX3 7LD, UK. ${ }^{2}$ MRC Lifecourse Epidemiology Unit, Southampton General Hospital, University of Southampton, Southampton SO16 6YD, UK. ${ }^{3}$ Faculty of Health Sciences, University of Southampton, Highfield, Southampton SO17 1BJ, UK. ${ }^{4}$ Nuffield Department of Primary Care Health Sciences, Radcliffe Observatory Quarter, Oxford OX2 6GG, UK. ${ }^{5}$ School of Clinical Sciences, University of Bristol, Learning and Research Building, Level 1, Southmead Hospital, Bristol BS10 $5 \mathrm{NB}, \mathrm{UK}$.

Received: 5 September 2014 Accepted: 7 April 2015

Published online: 23 April 2015

\section{References}

1. Marsh D, Currie C, Brown P, Cooper A, Elliott J, Griffiths R, et al. The care of patients with fragility fractures. British Orthopaedic Association; 2007.

2. Chesser TJS, Handley R, Swift C. New NICE guideline to improve outcomes for hip fracture patients. Injury. 2011;42(8):727-9.

3. Dennison E, Mohamed MA, Cooper C. Epidemiology of osteoporosis. Rheum Dis Clin North America. 2006;32(4):617-29. doi:10.1016/ j.rdc.2006.08.003

4. Cooper $C$, Mitchell $P$, Kanis J. Breaking the fragility fracture cycle. Osteoporos Int. 2011;22(7):2049-50. doi:10.1007/s00198-011-1643-9.

5. Abrahamsen B, van Staa T, Ariely R, Olson M, Cooper C. Excess mortality following hip fracture: a systematic epidemiological review. Osteoporos Int 2009;20(10):1633-50. doi:10.1007/s00198-009-0920-3.

6. Johnell O, Kanis JA, Odén A, Sernbo I, Redlund-Johnell I, Petterson C, et al. Fracture risk following an osteoporotic fracture. Osteoporos Int. 2004;15(3):175-9. doi:10.1007/s00198-003-1514-0.

7. George GHM, Patel S. Secondary prevention of hip fracture. Rheumatology. 2000;39(4):346-9

8. Melton L, Kearns A, Atkinson E, Bolander M, Achenbach S, Huddleston J, et al. Secular trends in hip fracture incidence and recurrence. Osteoporos Int. 2009;20(5):687-94. doi:10.1007/s00198-008-0742-8.

9. Scottish Intercollegiate Guidelines Network (SIGN). Management of hip fracture in older patients. Edinburgh: Scottish Intercollegiate Guidelines Network (SIGN); 2009. p. CG111.

10. National Institute for Health and Clincial Excellence (NICE). Falls: the assessment and prevention of falls in older people. Manchester: National Institute for Health and Clinical Excellence (NICE); 2013. p. CG161.

11. National Institute for Health and Care Excellence (NICE). Osteoporosis secondary prevention including strontium ranelate. Manchester: National Institute for Health and Care Excellence; 2008. p. TA161.

12. Akesson K, Marsh D, Mitchell PJ, McLellan AR, Stenmark J, Pierroz DD, et al. Capture the fracture: a best practice framework and global campaign to break the fragility fracture cycle. Osteoporos Int. 2013;24(8):2135-52. doi:10.1007/s00198-013-2348-z.

13. Marsh D, Åkesson K, Beaton D, Bogoch E, Boonen S, Brandi ML, et al. Coordinator-based systems for secondary prevention in fragility fracture patients. Osteoporos Int. 2011;22(7):2051-65. doi: 10.1007/s00198-011-1642-x.

14. McLellan A, Gallacher S, Fraser M, McQuillian C. The fracture liaison service: success of a program for the evaluation and management of patients with osteoporotic fracture. Osteoporos Int. 2003;14(12):1028-34. doi:10.1007/ s00198-003-1507-z.

15. Treml J, Husk J, Lowe D, Vasilakis N. Falling standards, broken promises: report of the national audit of falls and bone health in older people 2010. London: Royal College of Physicians; 2011.

16. Johansen A, Wakeman R, Boulton C, Plant F, Roberts J, Williams A. A National Hip Fracture Database: National report 2013. London: Royal College of Physicians; 2013.

17. Drew S, Sheard S, Chana J, Cooper C, Javaid MK, Judge A. Describing variation in the delivery of secondary fracture prevention after hip fracture: 
an overview of 11 hospitals within one regional area in England. Osteoporos Int. 2014;25(10):2427-33. doi:10.1007/s00198-014-2775-5.

18. Peters DH, Adam T, Alonge O, Agyepong IA, Tran N. Implementation research: what it is and how to do it. BMJ. 2013;347. doi:10.1136/bmj.f6753.

19. Eccles M, Mittman B. Welcome to implementation science. Implement Sci. 2006;1(1):1.

20. May C. Towards a general theory of implementation. Implement Sci. 2013:8:18. doi:10.1186/1748-5908-8-18.

21. Rogers EM. The diffusion of innovation 5th edition. New York: Free Press; 2003

22. Rogers EM. A prospective and retrospective look at the diffusion model. J Health Commun. 2004:9(sup1):13-9. doi:10.1080/10810730490271449.

23. Grol RP, Bosch MC, Hulscher ME, Eccles MP, Wensing M. Planning and studying improvement in patient care: the use of theoretical perspectives. Milbank Q. 2007;85(1):93-138. doi:10.1111/j.1468-0009.2007.00478.x.

24. Michie S, Johnston M, Abraham C, Lawton R, Parker D, Walker A. Making psychological theory useful for implementing evidence based practice: a consensus approach. Qual Saf Health Care. 2005;14(1):26-33. doi:10.1136/ qshc.2004.011155.

25. Mair FS, May C, O'Donnell C, Finch T, Sullivan F, Murray E. Factors that promote or inhibit the implementation of e-health systems: an explanatory systematic review. Bull World Health Organ. 2012;90(5):357-64. doi:10.2471/ blt.11.099424.

26. May CR, Finch TL, Cornford J, Exley C, Gately C, Kirk S, et al. Integrating telecare for chronic disease management in the community: what needs to be done? BMC Health Serv Res. 2011;11:131. doi:10.1186/1472-6963-11-131.

27. Murray E, Burns J, May C, Finch T, O'Donnell C, Wallace $P$, et al. Why is it difficult to implement e-health initiatives? A qualitative study. Implement Sci. 2011;6:6. doi:10.1186/1748-5908-6-6.

28. McEvoy R, Ballini L, Maltoni S, O'Donnell CA, Mair FS, Macfarlane A. A qualitative systematic review of studies using the normalization process theory to research implementation processes. Implement Sci. 2014;9:2. doi:10.1186/1748-5908-9-2.

29. Kennedy A, Rogers A, Bowen R, Lee V, Blakeman T, Gardner C, et al. Implementing, embedding and integrating self-management support tools for people with long-term conditions in primary care nursing: a qualitative study. Int J Nurs Stud. 51(8):1103-13. doi:10.1016/j.jijurstu.2013.11.008.

30. Kennedy A, Rogers A, Chew-Graham C, Blakeman T, Bowen R, Gardner C, et al. Implementation of a self-management support approach (WISE) across a health system: a process evaluation explaining what did and did not work for organisations, clinicians and patients. Implement Sci. 2014;9:129. doi:10.1186/s13012-014-0129-5.

31. Bamford C, Poole M, Brittain K, Chew-Graham C, Fox C, lliffe S, et al. Understanding the challenges to implementing case management for people with dementia in primary care in England: a qualitative study using normalization process theory. BMC Health Serv Res. 2014;14:549. doi:10.1186/s12913-014-0549-6.

32. Atkins S, Lewin S, Ringsberg KC, Thorson A. Provider experiences of the implementation of a new tuberculosis treatment programme: a qualitative study using the normalisation process model. BMC Health Serv Res. 2011;11:275. doi:10.1186/1472-6963-11-275.

33. Spangaro J, Poulos RG, Zwi AB. Pandora doesn't live here anymore: normalization of screening for intimate partner violence in Australian antenatal, mental health, and substance abuse services. Violence Vict. 2011;26(1):130-44.

34. Miles MB, Huberman AM. Qualitative data analysis: a sourcebook of new methods. Thousand Oaks, CA: Sage; 1994.

35. Atkinson R, Flint J. Sampling, snowball: accessing hidden and hard-to-reach populations. In: Miller RL, Bewer JD, editors. The A-Z of social research. London, England: SAGE Publications, Ltd; 2003. p. 275-81.

36. Saumure K, Given LM. Data saturation. In: Given LM, editor. The SAGE encyclopedia of qualitative research methods. Thousand Oaks, CA: SAGE Publications, Inc; 2008. p. 196-7.

37. Baker SE, Edwards R. How many qualitative interviews is enough? National Centre for Research Methods. 2012

38. Ayres L. Semi-structured interview. In: Given LM, editor. The SAGE encyclopedia of qualitative research methods. Thousand Oaks, CA: SAGE Publications, Inc; 2008. p. 811-2.

39. Shank G. Abduction. In: Given LM, editor. The SAGE encyclopedia of qualitative research methods. Thousand Oaks, CA: SAGE Publications, Inc; 2008. p. 2-3.

40. Ritchie J, Lewis J. Qualitative research practice: a guide for social science students and researchers. London: Sage; 2003.
41. Pope C, Ziebland S, Mays N. Analysing qualitative data. BMJ. 2000:320(7227):114-6. doi:10.1136/bmj.320.7227.114.

42. Mitchell PJ. Best practices in secondary fracture prevention: fracture liaison services. Curr Osteoporos Rep. 2013;11(1):52-60. doi: 10.1007/s11914-012-0130-3

43. Ganda K, Puech M, Chen JS, Speerin R, Bleasel J, Center JR, et al. Models of care for the secondary prevention of osteoporotic fractures: a systematic review and meta-analysis. Osteoporos Int. 2013;24(2):393-406. doi:10.1007/ s00198-012-2090-y.

44. Eisman JA, Bogoch ER, Dell R, Harrington JT, McKinney Jr RE, McLellan A, et al. Making the first fracture the last fracture: ASBMR task force report on secondary fracture prevention. J Bone Miner Res. 2012;27(10):2039-46. doi:10.1002/jbmr.1698.

45. Mason B, Epiphaniou E, Nanton V, Donaldson A, Shipman C, Daveson BA, et al. Coordination of care for individuals with advanced progressive conditions: a multi-site ethnographic and serial interview study. $\mathrm{Br} J \mathrm{Gen}$ Pract. 2013:63(613):e580-8. doi:10.3399/bjgp13X670714.

46. Bevan G, Janus K. Why hasn't integrated health care developed widely in the United States and not at all in England? J Health Polit Policy Law. 2011;36(1):141-64. doi:10.1215/03616878-1191135.

47. Stevens PE, O'Donoghue DJ. The UK model for system redesign and chronic kidney disease services. Semin Nephrol. 2009;29(5):475-82. doi:10.1016/ j.semnephrol.2009.06.004

48. National Collaboration for Integrated Care and Support. Integrated care and support: our shared commitment. London: Department of Health; 2013.

49. Limb M. NHS England slips further behind on its efficiency savings target. BMJ. 2013;347. doi:10.1136/bmj.f5297.

50. Monitor. Closing the NHS funding gap: how to get better value health care for patients. London: Monitor; 2013.

\section{Submit your next manuscript to BioMed Central and take full advantage of:}

- Convenient online submission

- Thorough peer review

- No space constraints or color figure charges

- Immediate publication on acceptance

- Inclusion in PubMed, CAS, Scopus and Google Scholar

- Research which is freely available for redistribution

Submit your manuscript at www.biomedcentral.com/submit 